\title{
Papyrologische Lesefrüchte
}

\author{
Dieter Hagedorn (Köln)
}

\section{BGU XIX 2761}

Beeidete Erklärung des Ibispflegers Leon gegenüber dem Strategen vom 19. Okt. 52 v.Chr., in der Leon sich zur Bestellung seines Lehenslandes verpflichtet. Nach der Versicherung, bei Pflichtverletzung die fälligen Abgaben aus eigenen Mitteln

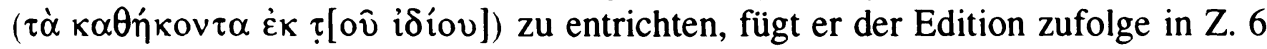

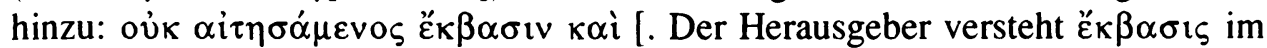
Sinne von „Entlassung aus einem Abkommen“ (s. Komm.) und übersetzt entsprechend „ohne um Entlassung und ... nachzusuchen“. Meines Erachtens steht im

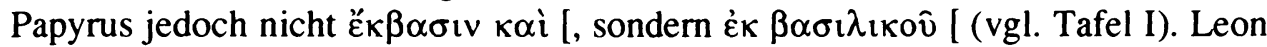
will hier wohl klarstellen, daß er vom Staat kein Saatdarlehen erbeten hat und daher auch nicht zur Rückzahlung eines solchen verantwortlich ist; ${ }^{1}$ man könnte in der folgenden Lücke also e.g. $\sigma \pi \varepsilon \dot{\varepsilon} \rho \alpha \tau \alpha$ ergänzen. Bei dieser Interpretation wird auch der Aorist $\alpha i$ i $\sigma \alpha \alpha \mu \varepsilon v o \varsigma$ ganz adäquat, während beim Verständnis des Herausgebers ein Präsens oder Futur natürlicher wäre.

\section{BGU XIX 2800}

In der fragmentarischen „Abrechnung über Hemden“ aus dem 7. Jh. n.Chr. liest man - unter der Überschrift $\gamma v(\hat{\omega} \sigma \mathrm{\iota}) \kappa \alpha \mu \iota \sigma i \omega v \dot{\alpha} \gamma o \rho \alpha \sigma \theta(\dot{\varepsilon} v \tau \omega v)-$ in Z. 2 von

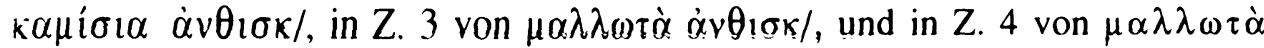

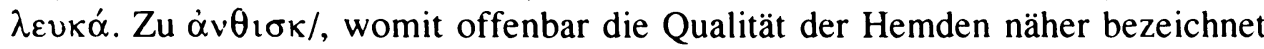
wird, wie besonders der Vergleich mit $\lambda \varepsilon v \kappa \alpha ́$ in Z. 4 nahelegt, erläutert der Herausgeber im Kommentar zu Z. 2-3, es handele sich um „ein bisher nicht belegtes Adjektiv, aufzulösen wohl zu $\dot{\alpha} v \theta i \sigma \kappa(\imath v \alpha)$, was zu dem ebenfalls nicht

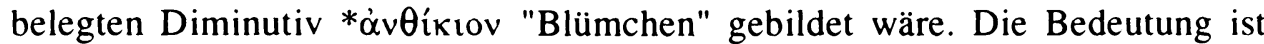
vermutlich $=\not \alpha v \theta \imath v \alpha$, was nicht unbedingt "geblümt" zu heißen braucht, sondern einfach "bunt" bedeutet"; im Wortregister auf S. 187 werden die Stellen dagegen

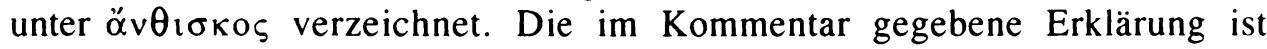
zweifellos denkbar. Ich frage mich indessen, ob es sich nicht eher um eine graphische Variante des in zeitlicher Nähe und in ähnlichem Zusammenhang, d.h.

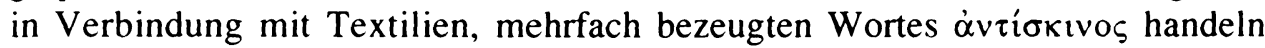

\footnotetext{
${ }^{1}$ Der Herausgeber vermutete hingegen: , $\tau \grave{\alpha}$ к $\alpha \theta \dot{\eta} \kappa o v \tau \alpha$ wird sich auf den Pachtzins ... und den Saatvorschuss beziehen" (Z. 6 Komm.).
} 
könnte. Die griechischen Belege dafür sind: P.Apoll. 104,13 (2. Hälfte 7. Jh.):

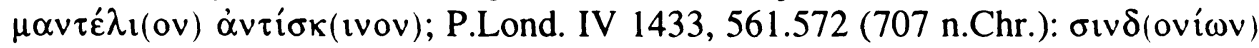
$\dot{\alpha} v \tau \imath \sigma \kappa i v(\omega v)$ und SB XVIII 13965,12 (6./7. Jh.): $\sigma ı v \delta$ (óvıov) $\dot{\alpha} v \tau i ́ \sigma \kappa(เ v o v)$. Ferner hatte schon Bell im Kommentar zu P.Lond. IV 1433,561 auf eine koptische Parallele aufmerksam gemacht: „Mr. Crum compares P.Rylands Copt. Pap.

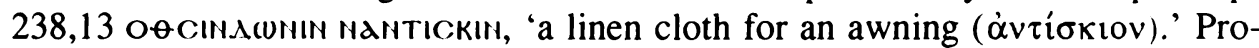
bably the article here is the same, and $\alpha \nu \tau \imath \sigma \mathrm{Kuv}^{-}$will represent an adjectival form

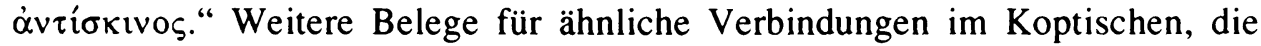
jedoch keine zusätzlichen Erkenntnisse bringen, zitiert jetzt Förster. ${ }^{2}$ Was die Herleitung und damit auch die Bedeutung des Wortes betrifft, so gehen die

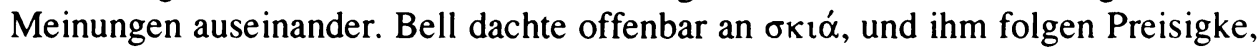
WB I 139 s.v. $\dot{\alpha} v i i ́ \sigma \kappa ı v \varsigma \varsigma$ mit der Wiedergabe „das Sonnensegel betreffend“, Diethart, der bei der Erstedition von SB XVIII 13965,12 in ZPE 64 (1986) 77 "Sonnensegeltuch" verwendet, und anscheinend auch Remondon, der in P.Apoll. 104,13 ,voile pour abriter“ übersetzt. Das „Revised Supplement“ von LSJ, das die Erstedition von SB XVIII 13965 zitiert, und Förster rechnen dagegen mit einem Itazismus und der Ableitung von $\sigma \kappa \eta v \eta ́$ (allerdings übernimmt Förster Preisigkes Übersetzung). Beide Deutungen überzeugen mich nicht recht, ohne daß ich allerdings einen anderen Vorschlag machen könnte.

\section{BGU XIX 2801}

Bei der Transkription der Kaisertitulatur in Z. 13-14 ist nach Tıßepíov versehentlich das Element K $\lambda \alpha v \delta$ íov ausgefallen (vgl. Tafel XIX). Es steht hier also dieselbe Formel wie in Z. 16-17, und es gibt kaum noch Anlaß zu der Vermutung, der Vertragsauszug von Z. 9-17 könne aus der Zeit des Tiberius statt der des Claudius stammen. Das Problem, das darin besteht, daß Claudius am 1. Okt. 40 (Z. 16-17) noch nicht den Thron bestiegen hatte, wird so zu lösen sein, wie schon F. Mitthof in APF 52 (2006) 271 erwogen hat: Beim Kopieren des Textes hat man wegen der abolitio nominis des Caligula den Wortlaut geändert.

\section{BGU XIX 2805}

Der Pachtvertrag des 5. Jh. n.Chr. betraf zwei Objekte, einmal eine größere Anzahl von Aruren, zum anderen eine einzige Arure. In Z. 7f., wo offenbar dieses

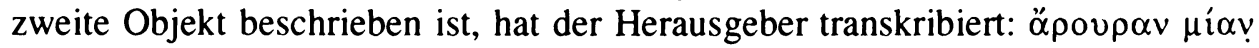

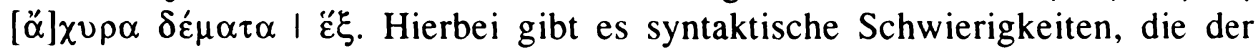
Herausgeber im Kommentar angedeutet hat. Nicht erwähnt aber hat er, daß man

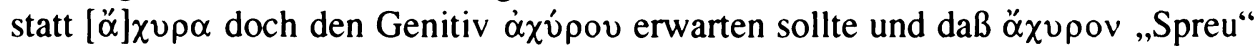

\footnotetext{
${ }^{2}$ H. Förster, Wörterbuch der griechischen Wörter in den koptischen dokumentarischen Texten (Texte und Untersuchungen zur Geschichte der altchristlichen Literatur 148), Berlin - New York

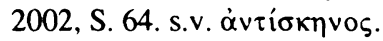


(nicht „Stroh“, wie übersetzt wird) nicht in „Bündeln“ gemessen werden kann (es gibt dafür in den Papyri auch keine Parallele). Tafel XXII hilft hier weiter; man

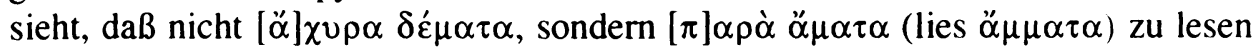
ist. Das zweite Objekt umfaßte also nur „eine Arure minus sechs Ammata“; man vergleiche die ähnliche Angabe in P.Herm. 62,5 (ebenfalls 5. Jh.): $\gamma \mathbf{i}(v \varepsilon \tau \alpha \imath)$ $(\not \alpha \rho o v \rho \alpha) \alpha \pi(\alpha \rho \grave{\alpha}) \not{\alpha} \mu(\mu \alpha \tau \alpha) \delta$. Eine Arure bestand aus 64 Ammata. $^{3}$

\section{BGU XIX 2828}

Bei der Subskription des Schreibhelfers des sich in dieser $\mu \iota \sigma \theta \alpha \pi \circ \chi \eta$ vom Anfang des 7. Jh. n.Chr. Verpflichtenden in Z. 13-14 fällt auf, daß er nicht das in dieser Zeit übliche Gentiliz Av̉ $\rho \dot{\eta} \lambda_{\text {ıo }}$ führt. Das spricht dafür, da $\beta$ es sich um einen Geistlichen handelt. ${ }^{4}$ In der Tat glaube ich auf Tafel XXXV am Ende von Z. 13 nach Namen und Vatersnamen, wo $\dot{\varepsilon} \lambda . . . .$. ( ) transkribiert wird, $\dot{\varepsilon} \lambda \alpha_{\alpha} \chi(\iota \sigma \tau \circ \varsigma)$ $\delta$ ıó́k(ovos) zu erkennen. In der unentzifferten Partie zu Beginn der nächsten Zeile, die ich auch nicht lesen kann, könnte dann eventuell der Name der Kirche zu suchen sein, in welcher der Diakon tätig war. Das erwartete Gentiliz hatte aber in jedem Fall der erste der Zeugen; denn am Ende von Z. 14 ist nach عỉótos $†$ in der Edition ein deutlich vorhandenes $\dagger A \cup \dot{p} \eta \dot{\lambda} \lambda$ เo dieser Stelle setzt daher schon die zweite Hand ein.

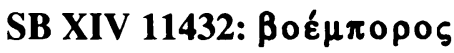

Der oxyrhynchitische Vertrag über den Verkauf eines Kalbes, den im späten 3. Jh. n.Chr. mehrere Verkäufer ${ }^{5}$ mit einem einzigen Käufer abgeschlossen haben, ist am Anfang und am Ende unvollständig. Er setzt nach der Lesung bzw. Interpretation der Herausgeberin ${ }^{6}$ ein mit dem Rest des Vatersnamens des letzten Verkäufers, worauf der Name und Vatersname der Käufers folge: $\Delta$ l]'lovṿgíov

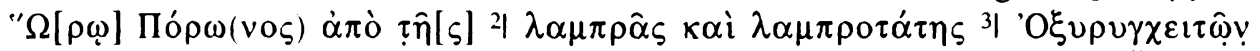
$\pi o ́ \hat{\imath} \varepsilon \omega s$. Der für den Vater des Käufers angesetzte Name Mó $\rho \omega v$ ist in Ägypten extrem selten; mir sind sonst nur noch die Belege in P.Heid. VI 374,104 und P.Lond. III 1227 (S. 143) Z. 5 und 7 bekannt. Bedenklich ist zudem, daß gerade ein so ungeläufiger Name abgekürzt worden sein soll, während sonst im Papyrus kaum Abkürzungen verwendet sind. Wie eine Abbildung, die im Internet eingesehen werden kann, ${ }^{7}$ zeigt, ist auch durch die Schreibweise - etwa durch Hochsetzung des letzten Buchstabens - nicht der geringste Hinweis auf eine

\footnotetext{
3 Vgl. nur J.C. Shelton, Land Measures in VBP IV 92, ZPE 42 (1981) 95-98; zuletzt CPR XXII, S. 166-169.

${ }^{4}$ Vgl. zuletzt K.A. Worp, On the Aureliate of Clergy and Monks, ZPE 151 (2005) 145-152.

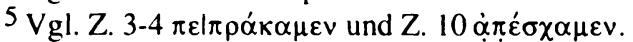

6 M.H. de Kat Eliassen, Five Papyri from the Oslo Collection, SO 49 (1973) 39-56, hier 50-52:

3. Sale of a Calf.

$7 \mathrm{Vgl}$. http://wwwapp.cc.columbia.edu/ldpd/app/apis/item?mode=item\&key=oslo.apis. 55.
} 
intendierte Abkürzung gegeben. Bedeutsamer ist aber eine durch die Abbildung ermöglichte zusätzliche Beobachtung: Wo die Herausgeberin das Omega von " $\Omega[\rho \omega]$ zu sehen glaubte, ist diese Lesung schwerlich möglich; zu spitz wäre die erste Rundung geraten, und die zweite Rundung müßte wie ein Omikron
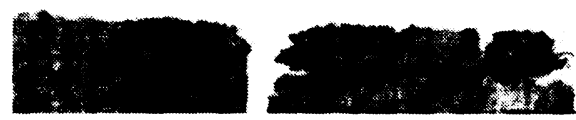
geschlossen sein. migen Typs und danach wirklich ein Omikron zu erkennen, und ergänze folglich zu $\beta o[\varepsilon \mu] \pi$ ó $\rho \varphi$. In $\Delta \mathrm{i}]$ loṿuóiov müssen wir daher den Vatersnamen nicht eines der Verkäufer, sondern des Käufers des Kalbes sehen, der passenderweise den Beruf eines ,Rinderhändlers“" ausübte, und da er nach Z. 11 wirklich ' $\Omega \rho \circ \varsigma$ hieß, muß man in Z. 0-1 ergänzen " $\Omega \rho \varphi \Delta \mathrm{t}]$ loṿvoíov $\beta o[\varepsilon \mu] \pi$ ó $\varphi$.

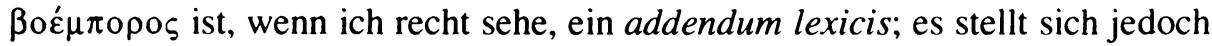

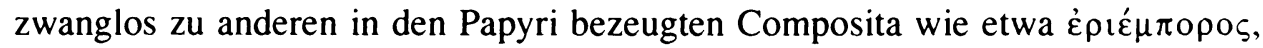

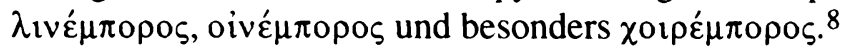

Zwei weitere Beobachtungen: In Z. 5 würde ich anstelle von $\pi \alpha \rho \alpha \tau \varepsilon \dot{\lambda} \eta \eta v$ (= $\pi \alpha \tau \rho \alpha \tau \varepsilon^{\prime} \varepsilon_{\varepsilon เ o v}$ ) die von der Herausgeberin ebenfalls erwogene Lesung $\pi \alpha \rho \alpha \tau \varepsilon^{\prime} \lambda_{\mathrm{l}} v$ bevorzugen. Am Ende von Z. 9 dürfte das notwendige Relativpronomen durch

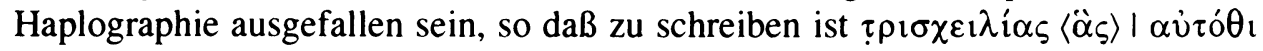
$\dot{\alpha} \pi \dot{\varepsilon} \sigma \chi \alpha \mu \varepsilon v$.

\section{SB XIV 12118}

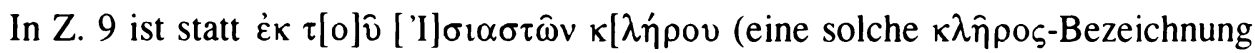
ist anderweitig nicht belegt) vermutlich die in Parallelen - d.h. Pachtverträgen des 4. Jh. n.Chr. aus dem Hermopolites - mehrfach bezeugte, aber noch nicht erklärte

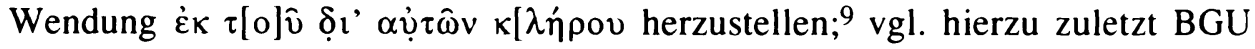
XIX 2802,4 mit den dort im Kommentar genannten Parallelen, dazu noch P.Münch. III 89,11f.

8 Vgl. H.-J. Drexhage, Einige Bemerkungen zu den $\check{\mu} \mu \pi$ o Ägypten (1.-3. Jh. n.), MBAH 10 (1991) 28-46, der feststellt, daß die traditionelle Unterscheidung

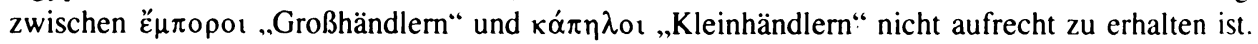
Eine Liste der bis 1991 aus den Papyri bekannt gewordenen Belege für $\ddot{\varepsilon} \mu \pi \circ \rho \circ \varsigma$ und dessen Composita findet sich dort in Appendix I, S. 40-42.

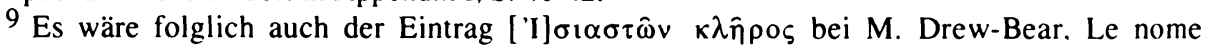
Hermopolite (ASP 21), Missoula 1979, S. 134, zu tilgen. 